\title{
Intramedullary spinal cord metastasis of malignant melanoma:Two cases with rim signs in contrast-enhanced magnetic resonance imaging: A case report
}

\author{
HARUKI MIZUTA $^{1,2}$, KENJIRO NAMIKAWA ${ }^{1}$, KENTA NAKAMA $^{1}$ and NAOYA YAMAZAKI ${ }^{1}$ \\ ${ }^{1}$ Department of Dermatologic Oncology, National Cancer Center Hospital, Tokyo 104-0045; \\ ${ }^{2}$ Department of Plastic and Reconstructive Surgery, Graduate School of Medicine \\ Osaka City University, Osaka 545-8586, Japan
}

Received April 28, 2020; Accepted September 17, 2020

DOI: $10.3892 / \mathrm{mco} .2021 .2209$

\begin{abstract}
Spinal cord metastasis of malignant melanoma is mostly caused by the invasion of the spinal cord by malignant melanoma. However, direct metastasis in the spinal cord is rare and difficult to diagnose accurately. A few diagnostically valuable findings of intramedullary spinal cord metastases (ISCMs) have been published. However, a highly specific finding of ISCMs of all carcinomas is the 'rim sign', which signifies the enhancement of the edge-dominant effect of the lesion in contrast-enhanced MRI. The objective of this case series was to examine the ratio of ISCMs of malignant melanoma with an indication of rim signs in contrast-enhanced MRI. The present report describes two cases of ISCMs of malignant melanoma in which the rim sign in contrast-enhanced MRI was useful for diagnosis.
\end{abstract}

\section{Introduction}

Most metastatic spinal cord tumors are epidural metastases caused by the direct invasion of vertebral metastatic tumors. Intramedullary spinal cord metastasis (ISCM) of malignant tumors is rare, accounting for $0.9-2.1 \%$ of all cases of spinal cord metastasis. Malignant melanoma accounts for approximately $9 \%$ of all cases of ISCM (1). A literature review was performed to include studies published in English on this topic, including our two cases. To the best of our knowledge,

Correspondence to: Dr Naoya Yamazaki, Department of Dermatologic Oncology, National Cancer Center Hospital, 5-1-1 Tsukiji, Chuo-ku, Tokyo 104-0045, Japan

E-mail: nyamazak@ncc.go.jp

Abbreviations: CT, computed tomography; ISCM, intramedullary spinal cord metastasis; MRI, magnetic resonance imaging

Key words: intramedullary spinal cord metastasis, malignant melanoma, rim sign, contrast-enhanced magnetic resonance imaging, immune-checkpoint inhibitor
27 cases of ISCMs associated with malignant melanoma have been reported; 12 cases were examined and 15 cases had no description of contrast MRI and were excepted. The rim sign was recognized in $33.3 \%$ (4/12) of these cases. However, none of the five cases of ISCMs of malignant melanoma identified in this series showed a rim sign. This study revealed that the rim sign is observed in ISCMs of malignant melanoma and in those of other cancers. We believe that the rim sign in an MRI is a clue for the diagnosis of ISCM of malignant melanoma.

\section{Case report}

Case 1. A 35-year-old woman with no significant medical history presented with malignant melanoma over the left clavicle. The tumor was $1.3 \mathrm{~mm}$ thick and had ulceration. Therefore, she was treated by resection of the primary lesion and axillary dissection. Metastases were detected in two of the 18 lymph nodes.

Four years later, she developed metastasis in the right lung, subcutaneous metastasis in the scalp, and multiple metastases in the bone. The tumors were positive for v-Raf murine sarcoma viral oncogene homolog B1 (BRAF) V600E mutation determined via a biopsy of metastasis in the scalp. Oral dabrafenib plus trametinib was initiated. Thereafter, all metastases reduced and her status was maintained. However, 5 years and 1 month after surgery, she developed multiple brain metastases and pleural dissemination and was treated with nivolumab and whole-brain irradiation. Bone metastases also progressed; therefore, the sternum and lumbar spine 5 were also irradiated. Four days after radiation therapy, she developed severe back pain. In addition, she experienced sensory disturbances caudal to the costal arch. She had no sensory disturbances or movement disorders in the upper limbs but had severe movement disorders in both lower limbs.

Contrast-enhanced magnetic resonance imaging (MRI) revealed a low-density mass with gadolinium-based contrast media at the peripheral ridge of T4-T6 (Fig. 1). Hence, an emergency evacuation was performed on the same day. Only a hematoma was intraoperatively visualized (Fig. 2A). However, although melanoma cells were not visualized, histopathological examination revealed that they were present, as evidenced 


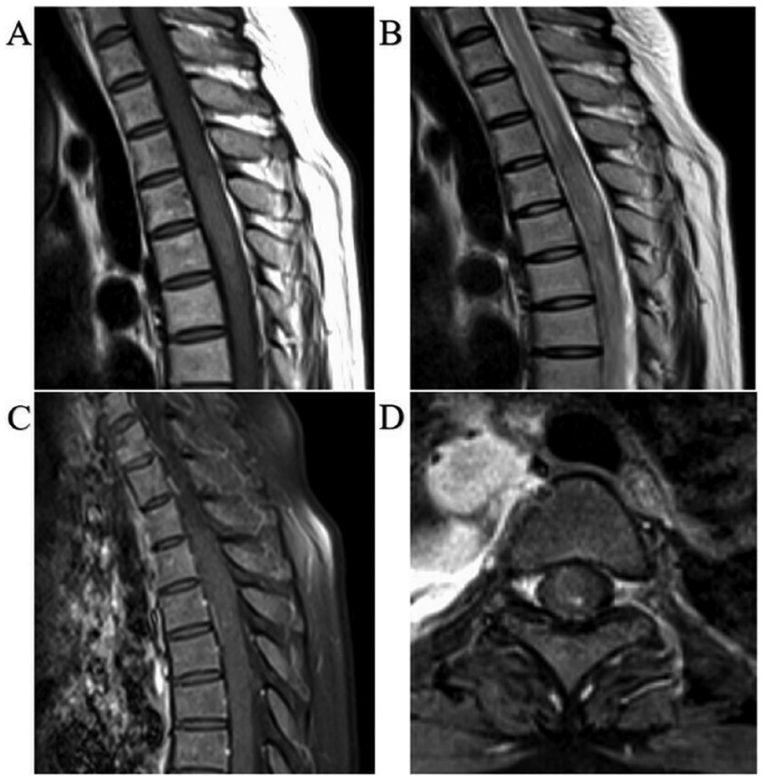

Figure 1. Magnetic resonance imaging of case 1 demonstrating the intramedullary spinal cord metastasis at the T6-T7 level. Rim signs were revealed after the administration of Gd-based contrast media. (A) Sagittal T2-weighted images. (B) Sagittal T1-weighted images. (C) Sagittal contrast-enhanced T1-weighted images. (D) Axial contrast-enhanced T1-weighted images.

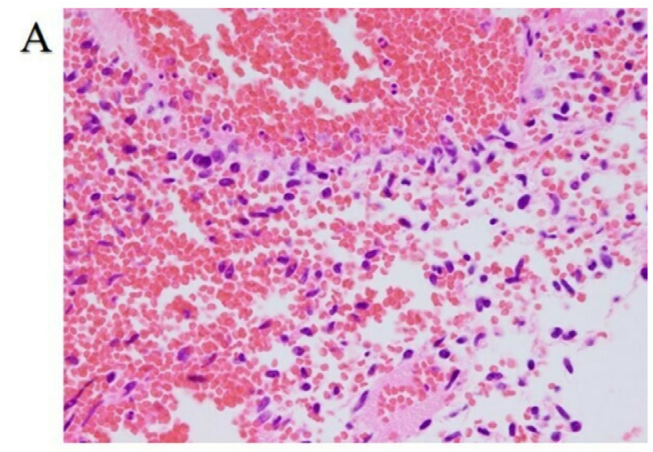

B

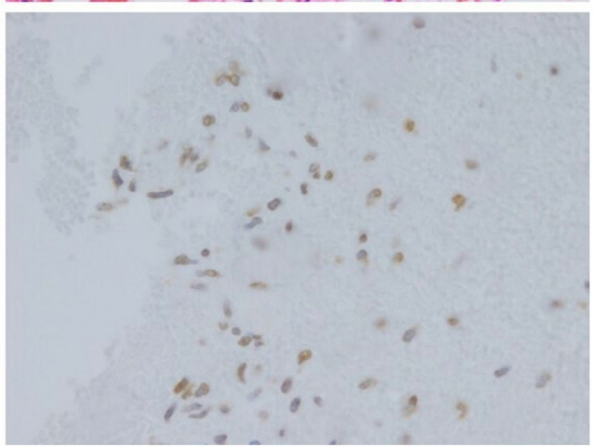

Figure 2. Histopathology. H\&E staining and IHC. (A) H\&E staining (magnification, $\mathrm{x} 400$ ). Melanoma cells could not be recognized. (B) IHC staining (magnification, $\mathrm{x} 400$ ). Tumor cells were positive for SOX-10. H\&E, hematoxylin and eosin; IHC, immunohistochemistry.

by SOX-10, S-100, and BRAF-V600E positive staining and the metastasis in the scalp (Fig. 2B). The patient was diagnosed with micro-metastases of malignant melanoma.

The day after surgery, a marked improvement in back pain was reported; however, movement disorders in both her lower limbs persisted. Fourteen days after surgery, computed tomography (CT) revealed exacerbation of pleural
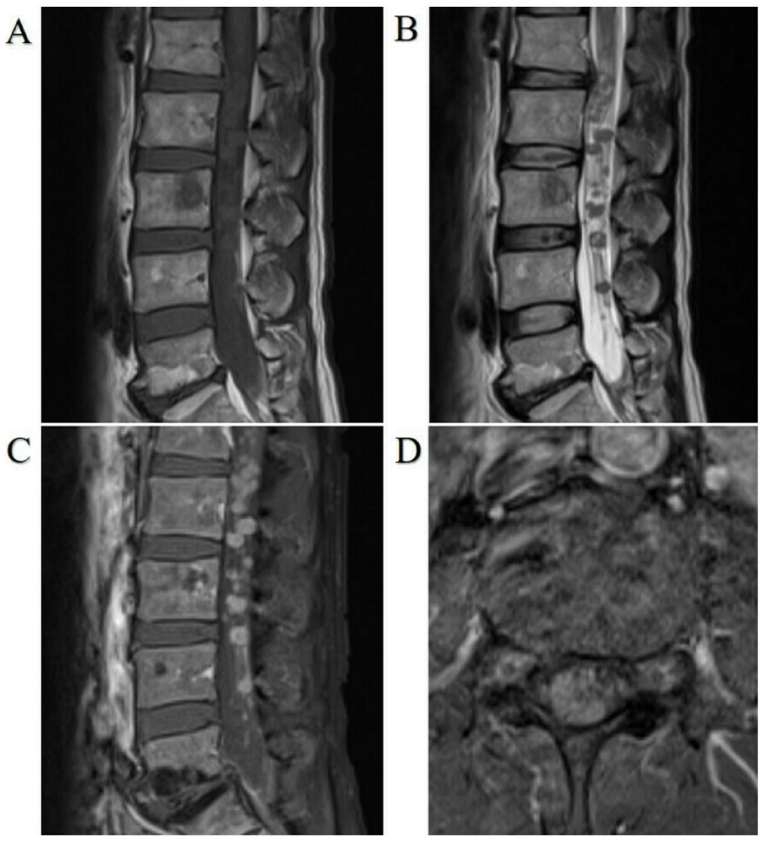

Figure 3. Magnetic resonance imaging of case 2 demonstrating the intramedullary spinal cord metastasis broadly at the lumbar level. Rim signs of multiple masses were revealed after the administration of Gd-based contrast media. (A) Sagittal T2-weighted images. (B) Sagittal T1-weighted images. (C) Sagittal contrast-enhanced T1-weighted images. (D) Axial contrast-enhanced T1-weighted images.

dissemination. Thereafter, oral dabrafenib (300 $\mathrm{mg}$ per day) plus oral trametinib ( $2 \mathrm{mg}$ per day) was resumed; it was highly effective in treating pleural dissemination. She developed fever; therefore, oral dabrafenib plus trametinib was continued subsequently. Because the antitumor effects of the treatment were apparent, cycles of treatment withdrawal and resumption were intermittently repeated. Six months after surgery, pleural dissemination increased in size. Therefore, nivolumab plus ipilimumab combination therapy was initiated. However, she died soon after.

Case 2. A 48-year-old man with a local recurrence of malignant melanoma of the left conjunctiva without BRAF mutation was treated by wide excision and reconstruction by a free flap. Because the stumps were partially positive, proton therapy was performed. Two months after the proton therapy, CT revealed right adrenal and retroperitoneal metastases. Therefore, nivolumab plus ipilimumab combination therapy was initiated. However, after 1 month, cervical spine and right upper humerus metastases were detected; thus, radiation therapy was performed. The dose was $20 \mathrm{~Gy}$ at $5 \mathrm{~Gy} /$ fraction. Eight months after the combination therapy, he experienced numbness and weakness in the right upper and lower limbs. Contrast-enhanced MRI revealed multiple masses with a contrast effect at the peripheral ridge of $\mathrm{C} 2$, Th1, and most of the lumbar spine (Fig. 3).

\section{Discussion}

Palliative radiation or conservative treatment is often selected for ISCM because the disease is associated with a poor prognosis. The main purpose of palliative radiation therapy is to 


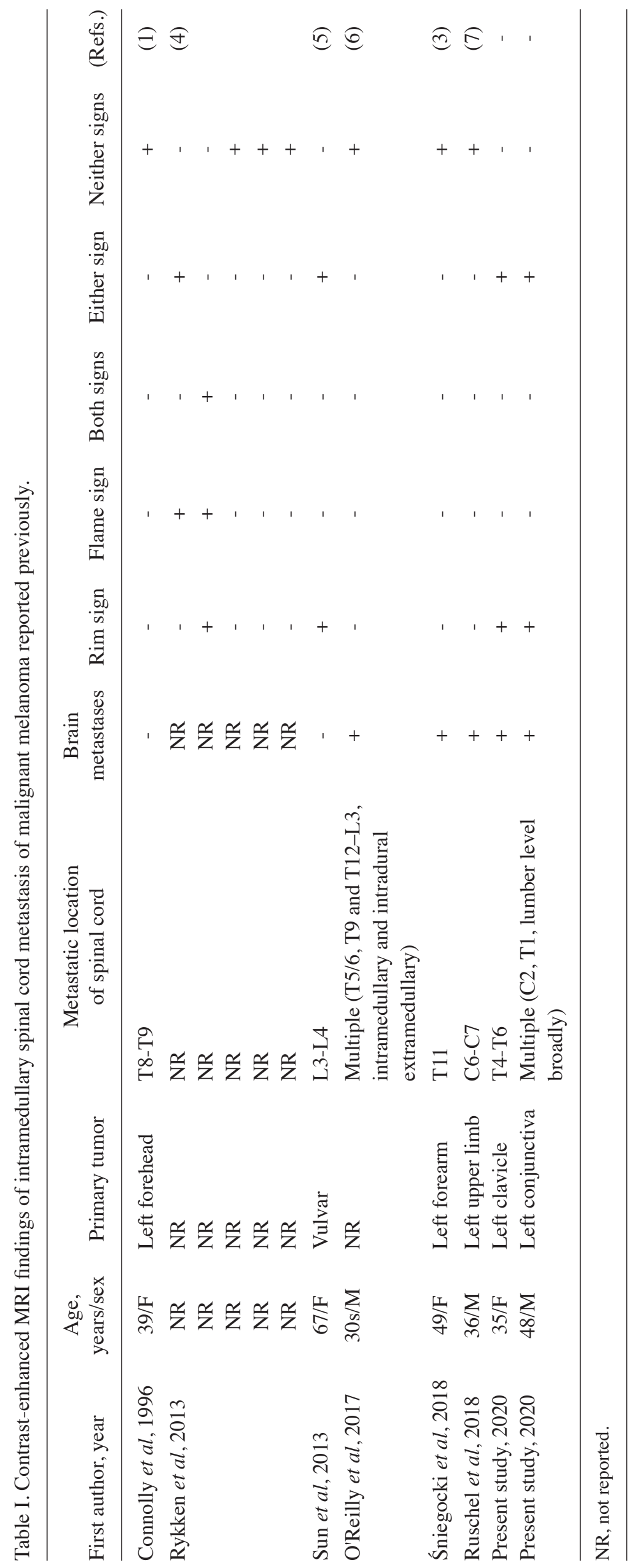


reduce the pain; therefore, radical cure cannot be achieved with only radiation therapy.

Patients expected to respond well to therapeutic intervention are considered candidates for surgical treatment, which can improve the overall survival and neurological functions (2). A few patients with ISCMs of malignant melanoma undergo surgical resection because melanoma tends to be highly resistant to radiation (3). Therefore, the dose of radiation for melanoma is often $4 \mathrm{~Gy}$ or more/fraction. In case 1 , surgical resection considerably reduced the patient's pain.

Considering the MRI findings, two unique characteristics of ISCMs, rim and flame signs, have been reported (4). Rim signs indicate a more intense thin rim with peripheral enhancement than other tumor areas and flame signs indicate flame-shaped enhancements at the edge of the lesion (above or below). These findings have been reported to be highly specific for ISCMs.

For all ISCMs, the rim sign was detected in $47 \%$ cases and the flame sign in $40 \%$ cases. Both signs were found in $27 \%$ cases and neither sign in $40 \%$ cases. Either sign was recognized in $60 \%$ cases. Melanoma was detected in five cases in this series but the rim sign was not detected in any $(0 / 5)$, whereas neither sign was observed in $60 \%$ cases $(3 / 5)(4)$. The authors of the respective studies did not mention these results because of the small number of cases of malignant melanoma.

According to a previous literature review, MRI could not reveal a specific pattern within the tumor owing to a mixture of melanin, intra-tumoral hemorrhage, and fat deposition (5). As a result, no characteristics have been reported so far to aid the diagnosis of ISCM of malignant melanoma.

To the best of our knowledge, 27 cases of ISCM of malignant melanoma (including our two cases) have been reported. In the literature, ISCM of malignant melanoma is described as a small percentage of the ISCMs of all cancer types; thus, it is sometimes not reported in detail.

We examined 12 cases, as the 15 other cases were excepted because there was no description of contrast-enhanced MRI findings (Table I) (5-7). One patient underwent MRI without using a contrast agent, but the characteristics of the lesion was described nonetheless (8).

Moreover, $83.3 \%$ (10/12) of these cases showed a single mass by MRI and multiple masses in only two cases (including our case 2). Another case was noted to have intramedullary and intradural extramedullary lesions.

Besides these two cases, a case with multiple masses discovered at autopsy has been reported (9). However, the lesions were not identified by an MRI in this case. Therefore, our case 2 is the first case of ISCM of malignant melanoma with multiple masses localized within the intramedullary area that was detected by MRI.

The rim sign was detected in $33.3 \%$ (4/12) and the flame sign was detected in $16.6 \%$ patients $(2 / 12)$. Both signs were found in $8.3 \%(1 / 12)$, either sign in $33.3 \%(4 / 12)$, and neither in $58.3 \%$ of patients (7/12). Although the ISCM of malignant melanoma was found in $33.3 \%$ patients, the rim sign frequency was slightly lower than that observed in the ISCM of other cancers. Conversely, the flame sign in ISCMs of malignant melanoma was less than that in ISCMs of other cancers.

In the cases of ISCMs of malignant melanoma, the incidence of brain metastasis was as high as $76.4 \%(13 / 17)$.
The response rate of intracranial metastases of BRAF V600 mutation-positive malignant melanoma to combination therapy with dabrafenib plus trametinib was $58 \%$ and that of extracranial metastases was 55\% (10). The response rate of intracranial lesions to the nivolumab plus ipilimumab combination therapy for BRAF V600 mutation-negative malignant melanoma was $57 \%$. Conversely, the response rate of intracranial metastases to immune-checkpoint inhibitor monotherapy was almost $22 \%$ (11). Hence, combination therapy was established to be significantly effective. Our cases are unique as patients with ISCMs of malignant melanoma are treated by immune-checkpoint inhibitors or molecule-targeted agents.

In conclusion, the rim sign is detected in some ISCMs of malignant melanoma and in ISCMs of other cancers. We believe that the rim sign in MRI is a useful diagnostic clue of ISCM of malignant melanoma. Although ISCM of malignant melanoma is difficult to diagnose accurately and is associated with poor prognosis owing to complications of brain metastases, the prognosis of malignant melanoma has substantially improved because of treatment advances. Therefore, more accurate diagnoses and the development of therapeutic strategies will help improve patients' quality of life in the future.

\section{Acknowledgements}

Not applicable.

\section{Funding}

The present study was supported by the National Cancer Center Research and Development Fund (grant no. 2020-J-3).

\section{Availability of data and materials}

The datasets used and/or analyzed during the current study are available from the corresponding author on reasonable request.

\section{Authors' contributions}

HM analyzed and interpreted the patient data and was a major contributor in writing the manuscript. NY and KNam offered valuable feedback regarding the study. KNak assisted in the early stages of this work. All authors read and approved the final manuscript.

\section{Ethics approval and consent to participate}

Not applicable.

\section{Patient consent for publication}

A waiver of informed consent requirement was obtained from the National Cancer Center Hospital Institutional Review Board.

\section{Competing interests}

The authors declare that they have no competing interests. 


\section{References}

1. Connolly JE Jr, Winfree CJ, McCormick PC, Cruz M and Stein BM: Intramedullary spinal cord metastasis: Report of three cases and review of the literature. Surg Neurol 46: 329-338, 1996.

2. Goyal A, Yolcu Y, Kerezoudis P, Alvi MA, Krauss WE and Bydon M: Intramedullary spinal cord metastases: An institutional review of survival and outcomes. J Neurooncol 142: 347-354, 2019.

3. Śniegocki M, Nowacka A, Smuczyński W and Woźniak K: Intramedullary spinal cord metastasis from malignant melanoma: A case report of a central nervous system secondary lesion occurred 15 years after the primary skin lesion resection. Postepy Dermatol Alergol 35: 325-326, 2018.

4. Rykken JB, Diehn FE, Hunt CH, Eckel LJ, Schwartz KM, Kaufmann TJ, Wald JT, Giannini C and Wood CP: Rim and flame signs: Postgadolinium MRI findings specific for non-CNS intramedullary spinal cord metastases. AJNR Am J Neuroradiol 34: 908-915, 2013.

5. Sun L, Song Y and Gong Q: Easily misdiagnosed delayed metastatic intraspinal extradural melanoma of the lumbar spine: A case report and review of the literature. Oncol Lett 5: 1799-1802, 2013.

6. O'Reilly MK, Sugrue G, Byrne D and MacMahon P: Combined intramedullary and intradural extramedullary spinal metastases in malignant melanoma. BMJ Case Rep 2017: bcr2017220031, 2017.
7. Ruschel LG, Ramina R, da Silva EB Jr, Cavalcanti MS and Duarte JFS: 5-Aminolevulinic acid fluorescence-guided surgery for spinal cord melanoma metastasis: A technical note. Acta Neurochir (Wien) 160: 1905-1908, 2018.

8. Conill C, Sánchez M, Puig S, Planas I and Castel T: Intramedullary spinal cord metastases of melanoma. Melanoma Res 14: 431-433, 2004.

9. Ishii $\mathrm{T}$, Terao $\mathrm{T}$, Komine $\mathrm{K}$ and Abe $\mathrm{T}$ : Intramedullary spinal cord metastases of malignant melanoma: An autopsy case report and review of the literature. Clin Neuropathol 29: 334-340, 2010.

10. Davies MA, Saiag P, Robert C, Grob JJ, Flaherty KT, Arance A, Chiarion-Sileni V, Thomas L, Lesimple T, Mortier L, et al: Dabrafenib plus trametinib in patients with BRAFV600-mutant melanoma brain metastases (COMBI-MB): A multi-cohort, open-label, phase 2 trial. Lancet Oncol 18: 863-873, 2017.

11. Konstantinou MP, Dutriaux C, Gaudy-Marqueste C, Mortier L, Bedane C, Girard C, Thellier S, Jouary T, Grob JJ, Richard MA, et al: Ipilimumab in melanoma patients with brain metastasis: A retro-spective multicentre evaluation of thirty-eight patients. Acta Derm Venereol 94: 45-49, 2014.

This work is licensed under a Creative Commons Attribution-NonCommercial-NoDerivatives 4.0 International (CC BY-NC-ND 4.0) License. 